Mini Review

\title{
Quality of life and its assessment methods in laryngeal cancer
}

\begin{abstract}
Quality of life (QoL) is defined as complete physical, social, and mental well-being, not just absence of disease. Surgery of laryngeal cancer surgery oftenly causes loss or impairment of the voice which results in diminsihed social integration of the patients. On the other hand side effects of radiotherapy and chemoradiotherapy such as xerostomia and general fatique may affect patient's psychiatric condition. Since the treatment of laryngeal cancer affects breathing, swallowing and speech functions, it is important to select appropriate treatment modality in order to increase QoL of these patients. Knowledge about the factors affecting the QoL may help physicians to undestand the patients 'needs and concerns and to prescribe physical and psychosocial rehabilitation, if necessary. There are many questionnaires to assess quality of life patients which should be considered and studied in future trials by head and neck surgeons. SF 36 and 12, EORTC (European Organisation for Research and Treatment of Cancer) QLQ C30, EORTC QLQ-H \& N35, Voice Handicap Index (VHI)30, Voice-related Quality of Life (VRQOL) Questionnaire and MDADI (MD Anderson Dysphagia Inventory) are the major assessment tools described in the literature. In this review, the current literature regarding the QoL and its assessment methods in laryngeal cancer is summarized.
\end{abstract}

Keywords: laryngeal cancer, quality of life, surgery
Volume 8 Issue 4 - 2017

\author{
Emel Tahir,' Tevfik Sözen² \\ 'Otolaryngology Clinic University of Health Sciences Diskapi \\ Yildirim Beyazit Training and Research Hospital, Turkey \\ ${ }^{2}$ Department of Otolaryngology Hacettepe University Schol of \\ Medicine, Turkey
}

\begin{abstract}
Correspondence: Emel Tahir, Otolaryngology Clinic, University of Health Sciences Dıșkapı Yıldırım Beyazıt Training and Research Hospital, Dıșkapı Yıldırım Beyazıt EAH KBB Kliniği D blok 4. kat Altındağ, Ankara, Turkey, Tel 00903 I 25962000 , Email emeltahir@hotmail.com
\end{abstract}

Received: May 05, 2017 | Published: September II, 2017
Abbreviations: QoL, quality of life; VHI, voice handicap index; VRQOL, voice-related quality of life; MDADI, md anderson dysphagia inventory; EORTC, european organisation for research and treatment of cancer

\section{Introduction}

Treatment of laryngeal cancer commonly affects several basic physiological functions, such as breathing swallowing, speech, and salivary function. Selection of treatment modality and survival among these cases are both related to clinical stage at presentation. In selected situations where the surgical and non surgical treatment options (radiation therapy and chemotherapy) have shown similar survival benefits, Quality of Life (QoL) may be determinant factor in deciding between these modalities. ${ }^{1}$ Although survival is the main interest concerning the patient's treatment, other parameters such as quality of life are important, when various treatments options are compared, such as surgery and chemoradiation. ${ }^{2}$

QoL measurements can be useful as a secondary outcome measurement for patients with laryngeal cancer. ${ }^{3}$ In this paper, we reviewed the current literature regarding the $\mathrm{QoL}$ and its assessment methods in laryngeal cancer.

\section{Discussion}

\section{Treatment options for laryngeal cancer}

Early stage laryngeal cancer is generally treated with radiotherapy, transoral laryngeal microsurgery and open partial surgery; advanced stage laryngeal cancer is treated with total laryngectomy and/ or chemoradiotherapy. All of these treatment modalities have multiple impacts on speech, swallowing and breathing. If organ preservation is preferred for treatment, side effects of radiotherapy and chemoradiotherapy such as xerostomia and general fatique may affect patients' psychiatric condition. ${ }^{4-6}$
It was recently recognized by the "International Head and Neck Scientific Group" that "avoiding surgery at the expense of losing the ability to swallow and/or speak with other modes of therapy cannot be considered a success". ${ }^{7}$ On the other hand; survival may not be the single issue for all patients. People after laryngeal cancer surgery oftenly experience loss or impairment of the voice which results in diminsihed social integration and general socialization. Also people after total laryngectomy are in danger of stigmatization and social exclusion. ${ }^{8,9}$

\section{Factors determine the treatment success}

i. Mortality- 5year survival

ii. Morbidity: Bleeding, Mucositis, Wight loss, Systemic side effects

iii. Voice quality

iv. Recurrence rate

v. Quality of life

vi. Cost- effectiveness

\section{Quality of life and its assessment tools}

The World Health Organization defines quality of life as complete physical, social, and mental well-being, and not just absence of disease. ${ }^{10}$

Patients with laryngeal cancer usually experience changes in important vital functions such as breathing and swallowing related to the disease and its treatment, resulting in a negative impact on their QoL ${ }^{11,12}$. The QoL of head and neck cancer patients deteriorates immediately after treatment so that the application of specific questionnaires is beneficial to measure QOL in these patients. In 1980, the European Organisation for Research and Treatment of Cancer (EORTC) was the first oncology making an effort on the 
development of a QoL instrument. ${ }^{13}$ Quality of life (QoL) research studies increasingly becoming popular in cancer clinical trials and clinicians have many tools to quickly asses the QoL. ${ }^{14-16}$

\section{Conditions affecting qol in patients with head and neck cancer}

a. Demographic features such as age, gender, educational level and marital status

b. Stage of the disease

c. Presence of any comorbid disease

d. Presence of tracheotomy or feeding tube

e. Chemoradiotherapy

f. Neck dissection

\section{Quality of life measurement tools that can be used in laryngeal cancer patients}

1. SF36 and SF 12 (Short Form 36 and Short Form 12)

2. EORTC QLQ C30 (European Organisation for Research and Treatment of Cancer Quality of Life Questionnaire)

3. EORTC QLQ-H \& N35 (European Organisation for Research and Treatment of Cancer Quality of Life Questionnaire for Head and Neck Cancer)

4. Voice Handicap Index (VHI)- 30

5. Voice-related Quality of Life (VRQOL) Questionnaire

6. MD Anderson Dysphagia Inventory (MDADI)

\section{Conclusion}

The field of oncology has been shifted over the last years. Preserving the quality of life has become an equally important medical objective as excising the tumor and increasing patient survival. QoL is an important health domain to consider, besides survival, when evaluating the success of laryngeal cancer treatments. Therefore, the cure of the disease itself is crucial but the QoL must also be considered to establish parameters of rehabilitation and support.

There are many questionnaires to assess quality of life patients which should be considered and studied in future trials by head and neck surgeons. They are importants tools to guide future therapeutic decisions. Also they may improve the communication between the patient and physician and gives an additional idea to the physician about patient's well-being.

\section{Acknowledgements}

None.

\section{Conflicts of interest}

The authors declare that there is no conflict of interest regarding this manuscript.

\section{Funding}

None.

\section{References}

1. Polat B, Orhan KS, Kesimli MC, et al. The effects of indwelling voice prosthesis on the quality of life, depressive symptoms, and self-esteem in patients with total laryngectomy. Eur Arch Otorhinolaryngol. 2015;272(11):3431-3437.

2. Shuman AG, Larkin K, Thomas D, et al. Patient Reflections on Decision Making for Laryngeal Cancer Treatment. Otolaryngol Head Neck Surg. 2017;156(2):299-304.

3. Bradley PJ, Mackenzie K, Wight R, et al. Consensus statement on management in the UK: transoral laser assisted microsurgical resection of early glottic cancer. Clin Otolaryngol. 2009;34(4):367-373.

4. Spielmann PM, Majumdar S, Morton RP. Quality of life functional outcomes in the management of early glottic carcinoma: a systematic review of studies comparing radiotherapy and transoral laser microsurgery. Clin Otolaryngol. 2010;35(5):373-382.

5. Nakayama M, Loccourreye O, Hosinger FC, et al. Functional organ preservation for laryngeal cancer: past, present and future. Jpn J Clin Oncol. 2012;42(3):155-160.

6. Warner L, Chudasama J, Kelly CG, et al. Radiotherapy versus open surgery versus endolaryngeal surgery (with or without laser) for early laryngeal squamous cell cancer. Cochrane Database Syst Rev. 2014;12:CD002027.

7. Strojan P, Haigentz M, Bradford CR, et al. Chemoradiotherapy vs. total laryngectomy for primary treatment of advanced laryngeal squamous cell carcinoma. Oral Oncol. 2013;49(4):283-286.

8. Rossi VC, Fernandes FL, Ferreira MA, et al. Larynx cancer: quality of life and voice after treatment. Braz J Otorhinolaryngol. 2014;80(5):403-408.

9. Rinkel RN, Verdonck-de Leeuw IM, van den Brakel N, et al. atientreported symptom questionnaires in laryngeal cancer: voice, speech and swallowing. Oral Oncol. 2014; 50(8):759-764.

10. Bjordal BK, Hammerlid E, Ahlner-elmqvist M. The WHO QOL Group (1994) What quality of life. World Heal. Forum. WHO, Geneva, USA. 1999.

11. Terrell JE, Ronis DL, Fowler KE, et al. Clinical predictors of quality of life in patients with head and neck cancer. Arch Otolaryngol Head Neck Surg. 2004;130(4):401-408.

12. Karlsen T, Sandvik L, Heimdal JH, et al. Health-related Quality of Life as Studied by EORTC QLQ and Voice Handicap Index Among Various Patients With Laryngeal Disease. J Voice. 2017;31(2):251.

13. Chaukar DA, Das AK, Deshpande MS, et al. Quality of life of head and neck cancer patient: validation of the European organization forresearch and treatment of cancer QLQ-C30 and European organization for research and treatment of cancer QLQ-H\&N 35 in Indian patients. Indian J . 2005;42(4):178-184.

14. Blanchard P, Volk RJ, Ringash J, et al. Assessing head and neck cancer patient preferences and expectations: A systematic review. Oral Oncol. 2016;62:44-53

15. Rogers SN, Semple C, Babb M, et al. Quality of life considerations in head and neck cancer: United Kingdom National Multidisciplinary Guidelines. J Laryngol Otol. 2016;130(S2):49-52.

16. Kucuk H, Kurnaz SC, Kutlar G. Treatment expectations and quality of life outcomes of patients with laryngeal cancer based on different treatment methods. Eur Arch Otorhinolaryngol. 2015;272(5):1245-1250. 